\section{JUAMI unites US, African students on studies of materials for energy}

\author{
www.columbia.edu/cu/juami
}

http://juami2012.blogspot.com

W hile they come from different research backgrounds and speak different languages, 53 advanced graduate students from the United States and East Africa discovered that they share a common interest in materials for sustainable energy. They met at the school of the Joint US-Africa Materials Institute (JUAMI), which was held in Addis Ababa, Ethiopia, in December 2012. Two goals of the school were for the participants to learn about the research cultures in one another's countries and to develop avenues for future collaboration.

On the first day of the school, Peter Green, co-organizer of JUAMI and professor at the University of Michigan, gave an overview of the current energy outlook worldwide. Although fossil fuels still dominate the scene, renewables are on the rise and Green spoke optimistically about the future. Other issues that were raised included the role of energy efficiency, the need to couple energy production from intermittent sources such as solar and wind to energy storage, and the extent to which implementing renewables in Africa can help alleviate poverty.
This inaugural group of studentsincluding participants from the United States (19), Ethiopia (15), Tanzania (6), Uganda (6), Kenya (5), South Africa (1), and Zambia (1) - worked collaboratively on projects that ranged from the analysis of data from solar cells that they built and operated to problem sets on topics covered during the lectures. On Day 3, for example, Yury Gogotsi of Drexel University gave a tutorial on supercapacitors, after which students formed groups of five or six participants who worked on problem sets related to supercapacitors.

Eric Isaacs, a graduate student at Columbia University, said that one nice aspect about JUAMI has been the opportunity to make connections between different energy technologies and the materials upon which they rely. $\mathrm{He}$ found a common thread to be "the crucial role of nanoscale features in topperforming materials for nearly all the different technologies covered at the school." Another common thread, said JUAMI co-organizer Sossina Haile, was the balancing act required for creating devices from high-purity materials while

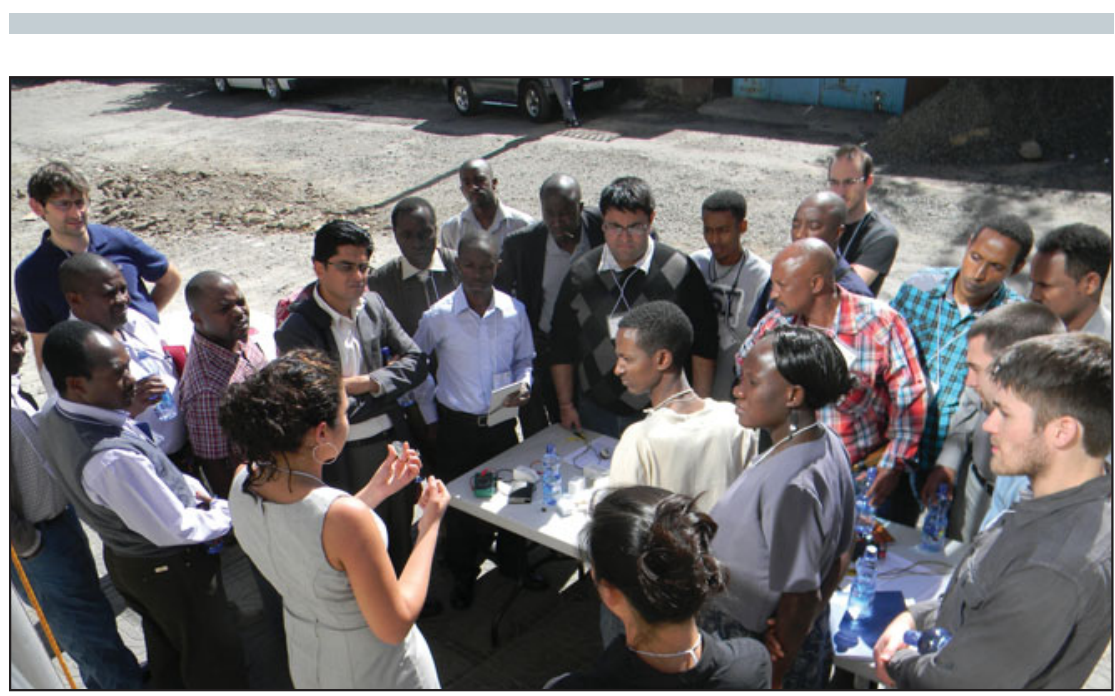

Students at the Joint US-Africa Materials Institute (JUAMI) work together to harvest electricity from the bright Ethiopian sun. Credit: Simon Billinge.

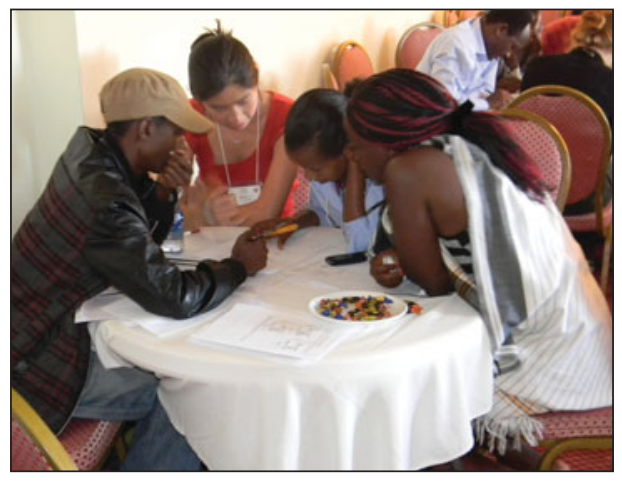

On Day 3 of JUAMI, participants break out into groups to solve problems related to supercapacitors, the topic of their tutorial earlier in the day. Credit: Simon Billinge.

maintaining low cost for market adoption, as highlighted in the new methods described for growing GaAs films for photovoltaic cells. Haile is a professor at the California Institute of Technology.

At the end of the workshop the students gave oral presentations on collaborative proposals they developed on topics that included conversion of local organic waste to mesoporous carbon for electrochemical energy storage and natural dye extraction for application in dye-sensitized solar cells. They also proposed a novel materials research exchange program for disseminating new findings to the participants, and development of computer codes for information exchange of technical challenges.

Now scientific collaborations involving various levels of information and personnel exchanges are planned. Also efforts are under way to establish JUAMI as a permanent institution, with the school to be held on an approximately biennial basis and rotating between various host countries.

The two-week school was supported primarily by a grant from the US National Science Foundation as proposed by Simon Billinge (Columbia University), Green, and Haile. They worked closely with an organizing committee from Africa, led by Teketel Yohannes (Addis Ababa University) and assisted by Bernard Aduda (University of Nairobi), Joseph Tesha (University of Dar Es Salaam), and Tom Otiti (Makerere University), to develop the curriculum and to select students. 